\title{
Multiscale and Multiobjective modelling: a perspective for mastering the design and operation complexity of IoT Systems
}

\author{
Khalil DRIRA \\ LAAS-CNRS, Université de Toulouse \\ CNRS, INSA, UPS, \\ Toulouse, France \\ khalil.drira@laas.fr
}

\begin{abstract}
Modeling IoT systems behaviors and architectures requires new approaches to be elaborated for handling the challenging issues such as: large scale interaction and real-time reconfiguration. This can help both during the design and the operation steps for ensuring a correct design and for delivering a performant service. Different studies have been recently conducted and new initiatives, at the national, European and international levels, have been taken for the domain of IoT systems and for the more general domain of System of Systems. Different modelling approaches have been elaborated. Bridging the models can lead to powerful solutions for modelling the next generation systems of systems of the family of IoT complex systems. Cross-disciplines initiatives have to be taken to enable the emergence of new Multiscale Multi-objective modelling approaches and frameworks.
\end{abstract}

Keywords-IoT, architecture; modelling; multiscale; systems-of-systems

\section{INTRODUCTION}

We tackle the complexity of architectural design for the Internet of Thing (IoT) complex systems and the future smart systems of systems built on top of IoT platforms. Internet of Thing platforms will connect billions of devices deployed on different geographic locations and manage different kinds of traffic generated by smart applications such as smart cities and connected vehicles. Design and modelling of such systems is a complex task that requires different levels of abstraction to be distinguished according to the properties to be validated. Recently, new architectural modelling techniques have been defined [4, 6]. These techniques handle the structural properties of communication architectures. The most used in IoT and distributed systems is the mediated communication pattern such as publish/subscribe brokers that decouple information producers and consumers, and load-balancers that distribute the load on different servers. Dynamic architecture modelling (e.g. graph-based dynamic structures), and analytic or quantitative approaches of behavioral performance modelling (e.g. Stochastic modelling, queueing theory) if integrated, can constitute a powerful modelling technique for a wide category of IoT systems. Such approaches address respectively, the problems of architectural reconfiguration strategies (service composition and deployment, network topology, etc.), and service provisioning policies (intelligent load balancing, resource allocation, etc.). The design of new integrated methods can be a promising objective that enables the elaboration of efficient multi-model solutions for self-configuring, self-healing, self-optimizing and self-protecting the IoT systems and the corresponding smart applications. Our objective, here, is to review the different studies and approaches in order to motivate the emergence of new modelling initiatives. We present the modeling challenges for Next Generation Systems in section II. We present the principles of multiscale modelling in section III. We consider the case of architectural models in section IV. We give a summary of our statements in section V.

\section{MOdeling Challenges FOR NG SyStemS}

Next Generation systems of systems such as IoT complex systems will be composed of a large number of interacting entities, possessing decision-making autonomy, and whose behavioral evolution is difficult to predict deterministically. A first cause may arise from the ignorance of certain parameters governing the evolution of the system or the random nature of their variation. These parameters may be intrinsic (internal variables), or context-dependent (external factors). Another cause may be the impossibility of performing, accurately or completely, within a reasonable time, the execution of the computation required to make it possible to determine the evolution of the state of the system. Communication networks (satellites, routers, gateways, protocol stacks), connected objects (sensors, actuators, devices, servers, appliances, machines, etc.) and intelligent services (discovery, security and protection, autonomy, etc.) as well as Smart Homes and Buildings, Smart Cities, Smart Power Grids, constitute an important part of the next generation complex systems that introduce important challenges for the design and operation steps. 
The modeling of the next generation complex systems is faced with scalability problems, heterogeneity of models, in particular structural and behavioral, and importance of both qualitative (correctness) and quantitative (performance or Time constraints). Elaborating the appropriate approaches can rely on modeling the evolution of a complex system by characterizing rather than enumerating or exploring all the configurations of the system architecture or its states space. To do this, models should be concerned with both the structural dimension and the behavioral (or functional) dimension of complex systems for analysis of correctness and performance properties.

Structural models have to be elaborated to describe a software architecture reconfigurable by dynamic integration of components or real-time composition of services. These models can also describe the topology of a network for which the graph represents the information propagation paths at various levels ranging from a representation of the neighborhood links in a sensor network to the relations of acquaintance in social networking or collaborative network platforms. The modeling can be based on conceptual graphs and graph grammars [2]. We can consider an initial graph and a set of transformation rules that characterize the set of the possible reachable configurations without their explicit enumeration. The transformation can operate by modifying the attributes associated with the nodes and the edges of the graph or by transforming its structure by adding or deleting nodes and arcs.

Behavioral models have to be elaborated to describe the functioning of the system. These models can describe the dissemination or the propagation of information according to an epidemic model, taking into account the influence of external factors of types interactions with users, and phenomenon of abandonment, etc. We can also proceed by a stochastic modeling of the system, the interactions between its components and its environment and we handle the scalability challenge through approximation models such as: fluid limits (e.g. for load balancing management) or the average field (e.g. for routing data in sensor networks). The challenge is to design systems where performance is associated with intelligence for the routing of information and its autonomous interpretation.

\section{Principles of Multiscale Modeling}

Multiscale modeling enables to look at a problem simultaneously from different scales and different levels of detail. It takes advantage of data available at distinct scales by modeling interaction between those scales, accordingly managing the complexity of behavior involved [3,7]. Practically this can be achieved by decomposing a problem into a set of single scale models that exchange information across the scales. In this context Borgdorff et al [1], gives a definition of a sub-model multiscale as a component model which describes only one scale of the system. He also considers a multiscale model as a composite model formed from two or more sub-models that describe different behaviors at different scales.

\section{A. Multiscale modeling strategies}

The key issue in multiscale modeling is the order in which the multiscale model is constructed. There are four strategies, discussed by [5], to establish such multiscale models:

- Bottom-up: Complex Systems can be understood on the higher scale by analyzing lower-scale mechanisms. A model is developed to describe the finest scale of interest, then models at increasing scales are constructed in turn; time or length scales may be used.

- Top-Down: A large scale model is constructed. It is refined by successively adding smaller scale models until detail and accuracy goals are reached.

- Middle-out: In some multiscale biological applications 'middle-out' modelling is favored. This refers to constructing a multiscale model by starting with the scales that are richest in data and best understood, and then working 'outwards' from there, to smaller and larger scales. level.

- Concurrent: All levels in the process hierarchy should be attacked simultaneously, from the microscopic level to the macroscopic

\section{B. Multiscale modelling steps:}

With the four strategies presented above, three steps are involved [5]:

- Step 1: identifying and selecting scales to include in the multiscale model

- Step 2: adopting or developing appropriate sub-models at each scale of interest

- Step 3: linking, or integrating, the sub-models into a coherent multiscale model. There are several broad ways of linking submodels into a multiscale model.

An important part of multiscale modeling is how the scales of different behaviors relate to each other. Multiscale simulation enables coupling of behaviors at various scales from the quantum scale to the molecular, mesoscopic, device, and plant scale [5].

Two approaches for linking sub-models at different scales are considered: sequential multiscale modeling and concurrent multiscale modeling.

With sequential multiscale modeling, the smallest (finest) scale model is solved first, and its results are passed to the larger (coarser) scale. For instance, some details of the macroscale model are precomputed using microscale models. 
Concurrent multiscale modeling is preferred when the macroscale model depends on many variables, and becomes difficult to extract by precomputing from microscale models. In addition, in some cases, the one-way coupling is inadequate, and fully coupled models across scales are needed, i.e., two-way information traffic exists. There are two types of multi-scale models:

\section{Multiscale Modeling Of Iot Systems}

Most problems in IoT are multiscale in nature. Things are made of sensors, actuators, gateways and servers at the atomic scale, and at the same time are characterized by their own architectural composition, geographic distribution as well as networking and processing capacities that have a larger order of magnitude.

\section{A. Scale concepts}

A scale is characterized by two major concepts: the grain and the extent. The grain is the finest spatial resolution; the resolution refers to the granularity used in the sub-system modelling. The extent refers to the structural or functional scope covered by the global system modelling. In the context of IoT systems modelling, the extent scale can refer to the abstract description considering a subsystem of the system architecture. Variation in extent can be used, for example, to describe a given description level or a given communication layer in the IoT infrastructure. It allows the architect to describe the necessary details to understand the system behavior and validate the associated functional and structural properties. Besides, the grain scale refers to the level of details and precision pertaining to the abstract description, providing more details of a given current description, such as composition and interactions in a given system description.

\section{B. Top-down scale transformations}

The top-down scale transformation process, much like regular refinement, begins with a high-level model of a system, which we describe as a whole. Then, scale changes are applied to obtain a more detailed description, by describing components that compose the subsystems and their connections. An iterative modeling allows to refine IoT systems descriptions: A vertical refinement can be applied to add the architecture composition details iteratively and to obtain a more detailed description by zooming on previously defined components. A horizontal refinement is needed to add details on the interconnections between components and their interfaces, and to establish the compatibility of interfaces by determining interfaces that can satisfy all possible sequences of required and provided relationships.

\section{CONCLUSION}

Multi-scale modelling of IoT systems architectures can be used to validate their behavioral properties both from the functional and non-functional points of views. We can consider static and dynamic structures with spatio-temporal properties. The abstraction or refinement related to grain and extent can address both components composition, routing or processing functions and exchanged data structure. The modelling strategies can be conducted in a bottom-up way allowing to characterize emerging properties in IoT systems viewed as systems of systems. We can also proceed by a top down modelling until we reach the necessary detail level for proving a given property such as information propagation in networked services.

\section{REFERENCES}

[1] J. Borgdorff, J.-L. Falcone, E. Lorenz, C. Bona-Casas, B. Chopard, and A. G. Hoekstra. Foundations of distributed multiscale computing: Formalization, specification, and analysis. Journal of Parallel and Distributed Computing, 73(4):465 - 483, Jan 2013.

[2] C. Eichler, T. Monteil, P. Stolf, L. Grieco, K. Drira. Enhanced graph rewriting systems for complex software domains. Software and Systems Modeling, Vol.15, N³, pp.685-705, July 2016, doi 10.1007/s10270-014-0433-1 ; 2015 Best papers award.

[3] W. Einer and B. Engquist. Multiscale modeling and computation. Notices Amer. Math. Soc, 50(50):1062-1070, 2003.

[4] A. Gassara, I. Bouassida, M. Jmaiel, K. Drira. A Bigraphical multi-scale modeling methodology for system of systems. Computers and Electrical Engineering, Vol.58, pp.113-125, Feb. 2017.

[5] G. Ingram, I. Cameron, and K. Hangos. Classification and analysis of integrating frameworks in multiscale modelling. Chemical Engineering Science, 59(11):2171-2187, 2004.

[6] I. Khlif, A. Hadj Kacem, A. Hadj Kacem, K. Drira. A multi-scale modelling perspective for SoS architectures. European Conference on Software Architecture ( ECSA ), Vienna (Austria) August 2014, LNCS 8627.

[7] E. Weinan and L. Jianfeng. Seamless multiscale modeling via dynamics on fiber bundles Communications in Mathematical Sciences. Volume 5, Issue 3 (2007), 649-663. 